\title{
Development of Modified Particles Method for Simulation of Debris Flow Using Constitutive Equations
}

\author{
Takuro SUZUKI ${ }^{1}$ and Norifumi HOTTA ${ }^{2}$ \\ ${ }^{1}$ Forestry and Forest Products Research Institute (1 Matsunosato, Tsukuba, Ibaraki 3058687, Japan) \\ E-mail: takurosuzuki@ffpri.affrc.go.jp \\ 2 Faculty of Life and Environmental Sciences, University of Tsukuba (1-1-1 Tennoudai, Tsukuba, Ibaraki 3058572, Japan)
}

\begin{abstract}
We propose a numerical simulation method for calculating the vertical distributions of the flow velocity and the sediment concentration in debris flows. Our method is based on the moving particle semi-implicit (MPS) method. We introduce the constitutive equations of Egashira et al. to the MPS method. Numerical simulations of the debris flow are performed by using an existing model based on a shallow water equation, along with our model based on the MPS method. In the condition where the riverbed gradient becomes less steep, there is good agreement with experimental results, including those involving the formation of a convex upward deposition shape in the initial deposition process. Results for the initial deposition process are not produced with existing simulation method based on a shallow water equation. Further, our model can yield clear results when the upper and lower layers have different flow directions in a numerical simulation of the collapse of a natural dam by overtopping.
\end{abstract}

Key words: debris flow, numerical simulation, moving particle semi-implicit method, constitutive equations

\section{INTRODUCTION}

Numerical simulations of debris flows are widely used to plan the locations for arrays of check dams [e.g., Nakagawa et al., 2001] and to calculate damage due to outbursts of landslide dams [e.g., Satofuka et al., 2007]. With these numerical simulations, the governing equations for the debris flow consist of the continuity equations and the momentum equation, both of which are based on shallow water equations. With these methods, the distributions of the flow velocity $u$ and sediment concentration $c$ are averaged vertically. Therefore, there is potential for a loss of accuracy in the calculations when the upper and lower layers have different flow directions.

Takahama et al. [2002] applied numerical simulation methods based on shallow water equations to a region around a point of an abrupt change in the gradient of the riverbed. They found that simulations of the bed profile provided good agreement with experimental results for advanced deposition processes; however, their numerical simulations were unable to reproduce the observed formations during convex upward deposition in the initial stages of the deposition process. This is thought to be due to vertical averaging of the flow velocity and sediment concentration, because the upper and lower layers can have different flow directions during the initial stages of the process. Constitutive equations [Egashira et al., 1989; 1997] can be used to reproduce the vertical distribution of the flow velocity and sediment concentration; however, existing simulation methods cannot fully implement these equations.

For these reasons, a method of calculating the vertical distribution of the flow velocity and sediment concentration is expected to be effective in reproducing such processes. Particle methods such as smooth particle hydrodynamics (SPH) [Muller et al., 2003] and the moving particle semi-implicit (MPS) method [Koshizuka and Oka, 1996] are considered appropriate for this purpose. The use of SPH and MPS methods has been reported for debris flows [e.g., Minatti and Pasculli, 2011; Abe et al., 2011]; however, these methods cannot reproduce the vertical distribution of the sediment concentration. 
In this study, we describe a modified MPS method for debris flows, whereby we introduce the constitutive equations of Egashira et al. [1989; 1997] to the standard MPS method [Koshizuka and Oka, 1996; Khayyer and Gotoh, 2008]. Moreover, numerical simulations using the existing method and the method provided here are described for deposition processes with an abrupt change in the gradient of the riverbed [Takahama et al., 2002] as well as the erosion process of natural landslide dam [Oda et al., 2010]. Finally, we discuss the applicability of the modified MPS method.

\section{MODIFIED MPS METHOD FOR DEBRIS FLOWS}

\subsection{Standard MPS method}

In this section the MPS method is briefly reviewed. A more detailed description can be found in Koshizuka and Oka [1996]. The governing equations are the continuity equation and Navier-Stokes equation, which describe the motion of a viscous incompressible fluid; i.e.,

$$
\frac{D \rho}{D t}=0
$$

and

$$
\frac{D \mathbf{u}}{D t}=-\frac{1}{\rho} \nabla P+v \nabla^{2} \mathbf{u}+\mathbf{g}
$$

where $\rho$ is the density of the fluid, $P$ is pressure, $\mathbf{u}$ is the velocity vector of the particles; $v$ is the laminar kinematic viscosity, and $\mathbf{g}$ is the acceleration due to gravity. These equations are discretized as follows using a particle model to describe the interaction between particle $i$ and its neighboring particles $j$, namely the gradient and Laplacian operators. The gradient operator is given by

$$
\nabla \phi_{i}=\frac{D_{0}}{n_{0}} \sum_{j \neq i}\left[\frac{\phi_{j}-\phi_{i}}{\left|\mathbf{r}_{j}-\mathbf{r}_{i}\right|^{2}}\left(\mathbf{r}_{j}-\mathbf{r}_{i}\right) w\left(\left|\mathbf{r}_{j}-\mathbf{r}_{i}\right|\right)\right]
$$

and the Laplacian operator is given by

$$
\nabla^{2} \phi_{i}=\frac{D_{0}}{m_{0}} \sum_{j \neq i}\left[\left(\phi_{j}-\phi_{i}\right) w\left(\mid \mathbf{r}_{j}-\mathbf{r}_{i}\right)\right]
$$

where $\phi$ is an arbitrary function, $D_{0}$ is the number of spatial dimensions, $n_{0}$ is the standard particle number density, $\mathbf{r}$ is the coordinate vector of the particle, $\gamma$ is a parameter that is introduced to fix the increase of the variance due to the re-distribution of the above equation equal to the increase in the variance estimated from the unsteady diffusion equation, and $w(r)$ is the kernel function; with the MPS method, the following kernel function is applied:

$$
w(r)=\left\{\begin{array}{cc}
\frac{r_{e}-1}{r} & \left(r \leq r_{e}\right) \\
0 & \left(r>r_{e}\right)
\end{array}\right\}
$$

where $r_{e}$ is the radius of influence of the particles, and is computed by multiplying the constant inter-particle distance $l_{0}$ by an arbitrary constant number.

The MPS method contains both explicit and implicit processes. With the explicit process, the tentative particle velocity vector $\mathbf{u}^{*}$ and the coordinate vector of the particle $\mathbf{r}^{*}$ are calculated using the viscous term and gravity term in Eq. (2); i.e.,

and

$$
\mathbf{u}^{*}=\mathbf{u}(t)+\Delta t\left\lfloor v \nabla^{2} \mathbf{u}(t)+g\right\rfloor
$$

$$
\mathbf{r}^{*}=\mathbf{r}(t)+\Delta t \mathbf{u} *
$$

where $\Delta t$ is the time step for the calculation. The terms $\mathbf{u}(t)$ and $\mathbf{r}(t)$ are the particle velocity vector and the coordinate vector of the particle at time $t$, respectively.

With this process, neither conservation of mass nor the incompressibility of the fluid are satisfied; hence, the intermediate particle velocities are updated by solving the Poisson equation for pressure, which describes the deviation in the particle number density. Via this implicit process, the corrected MPS (CMPS) method [Khayyer and Gotoh, 2008] is arrived at. The Poisson equation for pressure is as follows [Koshizuka et al., 1995]:

$$
\nabla^{2} P(t+\Delta t)=-\frac{\rho}{\Delta t^{2}} \frac{n_{i}-n_{0}}{n_{0}}
$$

where

$$
\left.n_{i}=\sum_{i \neq j} w\left(\mid \mathbf{r}_{j}-\mathbf{r}_{i}\right)\right)
$$

is the particle number density following the explicit process. The particle velocity vector and coordinate vector are updated using the resulting $P$; i.e.,

$$
\begin{gathered}
\mathbf{u}^{\prime}=-\frac{\Delta t}{\rho_{0}} \nabla P(t+\Delta t) \\
\mathbf{u}(t+\Delta t)=\mathbf{u}^{*}+\Delta t \mathbf{u}^{\prime}
\end{gathered}
$$

and

$$
\mathbf{r}(t+\Delta t)=\mathbf{r}^{*}+\Delta t \mathbf{u}^{\prime}
$$

\subsection{Debris flow shear stress model}

The modified MPS method for debris flows (MPS-DF) [Suzuki and Hotta, 2015] is arrived at by introducing the constitutive equations of Egashira et al., [1989; 1997]. The coordinate system is composed of an axis parallel to the riverbed, as well as an $\zeta$-axis perpendicular to the riverbed. The shear 
stress at a distance from bed $\tau(\zeta)$ is expressed as follows [Egashita et al., 1989; 1997]:

$$
\tau(\zeta)=\tau_{y}+\rho_{w} f(c)\left|\frac{\partial u}{\partial \zeta}\right|\left(\frac{\partial u}{\partial \zeta}\right)
$$

where

$$
\begin{array}{r}
\tau_{y}=\left(\frac{c}{c_{*}}\right)^{\frac{1}{5}} \rho_{w}\left(\sigma / \rho_{w}-1\right) g \cos \theta_{b} \\
\tan \phi_{s} \int_{\zeta}^{h} c d \zeta
\end{array}
$$

is the Coulomb friction shear stress, $\rho_{w}$ is the density of water, $c$ is the sediment concentration, $\sigma$ is the density of sedimentary particles, $\phi_{s}$ is the inter-particle friction angle, $h$ is the height of the flow, $\theta_{b}$ is the inclination of the riverbed, and

$$
f(c)=\left(K_{d}+K_{f}\right) d^{2}
$$

is the coefficient of resistance, where

$$
K_{d}=k_{g}\left(1-e^{2}\right)\left(\frac{\sigma}{\rho_{w}}\right) c^{\frac{1}{3}}
$$

and

$$
K_{f}=k_{f} \frac{(1-c)^{\frac{5}{3}}}{c^{\frac{2}{3}}}
$$

and where $d$ is the diameter of the sediment particles, $e$ is the coefficient of restitution, and where $k_{g}=$ 0.0828 and $k_{f}=0.16$ are empirical constants.

The above equations were derived under steady-state conditions; hence the shear stress is parallel to flow direction. With this method, these equations are extrapolated into unsteady conditions, as well as the existing simulation model, based on the shallow-water equation. For this reason, the shear stress term for the MPS-DF method is derived by differentiating Eq. (13) with respect to $\zeta$; i.e.,

$$
\begin{aligned}
\tau_{c}=\tau_{c y}+\rho_{w} \frac{\partial f(c)}{\partial c} \frac{\partial c}{\partial \zeta}\left|\frac{\partial u}{\partial \zeta}\right|\left(\frac{\partial u}{\partial \zeta}\right) \\
+2 \rho_{w} f(c)\left|\frac{\partial u}{\partial \zeta}\right|\left(\frac{\partial^{2} u}{\partial \zeta^{2}}\right)
\end{aligned}
$$

where

$$
\tau_{c y}=\left[\begin{array}{r}
\left.\frac{1}{5}\left(\frac{c}{c_{*}}\right)^{-\frac{4}{5}} \frac{\partial c}{\partial \zeta} h_{d} \cdot \bar{c}_{i}-\left(\frac{c}{c_{*}}\right)^{\frac{1}{5}} c\right] \\
\rho_{w}\left(\sigma / \rho_{w}-1\right) g \cos \theta \tan \phi_{s}
\end{array}\right.
$$

and

$$
\bar{c}_{i}=\int_{\zeta}^{h} c(\zeta) d \zeta /(h-\xi)
$$

and where $h_{d}$ is the difference in the $\zeta$-coordinate between particle $i$ and its free surface particle, $\bar{c}_{i}$ is the average sediment concentration of particles above particle $i$. MPS-DF is based on a Cartesian coordinate system containing gravity axis ( $Z$-axis). $\theta$ is the angle between the particle velocity vector and horizontal vector. The scalar quantity $\tau_{c}$ is converted to a vector $\boldsymbol{\tau}_{\mathbf{c}}$ using a rectangular coordinate system. The Navier-Stokes equation describing the motion of the debris flow is obtained by replacing the viscous term in Eq. 2 with $\tau_{\text {c }}$; i.e.,

$$
\frac{D \mathbf{u}}{D t}=-\frac{1}{\rho} \nabla P+\frac{\boldsymbol{\tau}_{\mathbf{c}}}{\rho}+\mathbf{g}
$$

\subsection{Sediment concentration}

A method of calculating the sediment concentration was developed because the shear stress term is a function of the sediment concentration $c$. In our model, the equilibrium sediment concentration of Egashita et al., [1997] is applied; i.e.,

$$
\begin{gathered}
(h-\zeta) \frac{\partial F}{\partial c} \frac{\partial c}{\partial \zeta}=F-c \\
\frac{\left(\frac{\sigma}{\rho_{w}}-1\right)}{(h)} K_{p d} \tan \theta \\
{\left[1-\left(\frac{c}{c_{*}}\right)^{\frac{1}{5}}\right]\left(K_{f}+K_{d}\right)-\left[\tan \theta-\tan \phi_{s}\left(\frac{c}{c_{*}}\right)^{\frac{1}{5}}\right] K_{p d}} \\
K_{p d}=k_{g} e^{2}\left(\frac{\sigma}{\rho}\right) c^{\frac{1}{3}}
\end{gathered}
$$

In the above equations, the equilibrium vertical distribution of $c$ is obtained by integrating the rate of change of $c$ in the vertical direction from the riverbed; i.e., $\partial c / \partial \zeta$. Replacing $\zeta$ with $Z$, these equations are applied to a Cartesian coordinate system. This conversion have no influence under steady-state conditions. However it may have a small influence under highly unsteady condition. This issue will be the subject of future work. With our method, $c$ moves among the neighboring particles, which can reduce the difference between the equilibrium range of change of $c$ and the actual rate of change of $c$. First, the equilibrium $\partial c / \partial Z$ is calculated using Eq. (22); i.e., $g c_{e}$, and present $\partial c / \partial Z$ is calculated using Eq. (3); i.e., gc. Figure 1 shows the concept of the variable sediment concentration model. Changes in the sediment concentration of an arbitrary particle are calculated for the underlying particles, because the distribution of $c$ is obtained by 


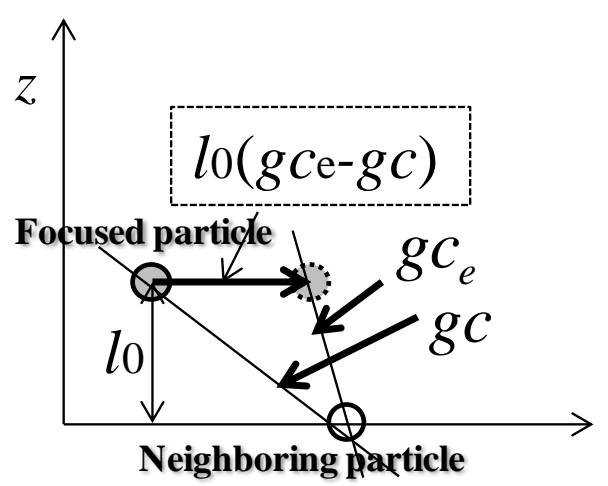

Fig.1 A schematic diagram illustrating the concept of the variable sediment concentration model.

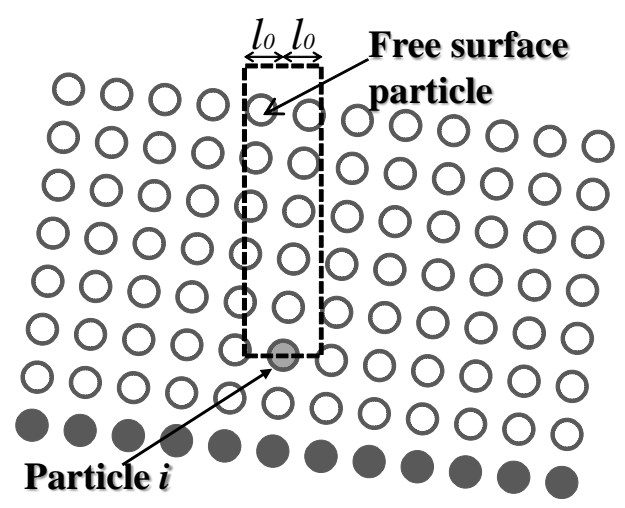

Fig. 2 The process used to calculate the free surface particles and average sediment concentration.

integrating $\partial c / \partial Z$ in the vertical direction from the riverbed. The particle shown in Fig. 1 will tend to result in an increase in $c$ because $g c_{e}>g c$. Therefore, $c c p(i)$, which expresses the magnitude and direction of change of $c$, is defined as follows:

$$
\operatorname{ccp}(i)=l_{0}\left(g c_{e}-g c\right)
$$

The time-derivative of $c$ is derived using kernel function (Eq. (5)), assuming that $c$ changes in proportion to the difference in $\operatorname{ccp}(i)$ between particles $i$ and $j$; i.e.,

$$
\left(\frac{\partial c}{\partial t}\right)_{i}=\frac{1}{T} \frac{1}{n_{0}} \sum_{i \neq j} w(r) \frac{1}{2}(c c p(i)-c c p(j))
$$

where $T$ is the relaxation time, and a long $T$ corresponds to highly non-equilibrium dynamics. In this study, a constant value of $T$ was applied because the theoretical interpretation thereof is not straightforward.

With MPS-DF, the process of calculating the sediment concentration is carried out after the implicit process.

\subsection{Method for calculating free surface particle and average sediment concentration}

The values of $h_{d}$ and $\bar{c}(Z)$ are required to evaluate Eqs. (19), (20) and (22). Figure 2 shows the process used to calculate the free surface particle and average sediment concentration. A free surface particle corresponding to particle $i$ is that particle which is located the highest among particles within a radius of $l_{0}$ in the horizontal direction. Here, $\bar{c}(Z)$ is the average of $c$ for particles within a radius of $l_{0}$ in the horizontal direction and located higher than particle $i$.

\subsection{Sediment layers}

Eq. (22) describes the flow layer and should not be applied to sediment layers. The sediment concentration of sediment layers is constant; that is, $\partial c / \partial Z=0$. For this reason, a transition region between the fluid and the deposited layer should be considered with equilibrium sediment concentrations $c_{e}$ and $\bar{c}(Z)$; i.e.,

$$
c_{e}=\frac{\rho_{w} \tan \theta}{(\sigma-\rho)\left(\tan \phi_{s}-\tan \theta\right)}
$$

This is one of two requirements used to determine whether a region will transition to a sediment layer with $\bar{c}(Z)>c_{e}$. With only this condition, instantaneous changes in $c$ might result in computational instabilities; hence, a second requirement is introduced: the average of $c$ of all particles within a radius of $l_{0}$ that are lower than particle $i$ should be larger than a threshold. Particles with a sediment concentration $>0.9 c *$ are considered to be deposited (i.e., the threshold is $\left.0.9 c^{*}\right)$. It follows that lower-lying particles to particle $i$ will form sediment layers. When a particle meets these two requirements, $g c_{e}=0$. The threshold value is not so dominant parameter. However, numerical oscillation occurs under a very low or very high threshold value, where $0.9 c *$ is a moderate value [Suzuki and Hotta, 2015].

\section{ONE DIMENTIONAL NUMERICAL SIMULATION}

In this section, we describe a one-dimensional (1D) numerical simulation based on the shallow water equation (hereinafter termed 1D-sim). The basic debris flow equations are shown below [Miyamoto and Itoh, 2002]. The momentum equation is

$$
\frac{\partial M}{\partial t}+\frac{\partial(u M)}{\partial x}=-g h \frac{\partial H}{\partial x}-\frac{\tau_{0}}{\rho_{m}}
$$

the continuity equation for the total volume of the debris flow is 
Table 1 The parameters used in the simulations.

\begin{tabular}{l|c}
\hline & Case 1 \\
\hline relaxation time, $T(\mathrm{~s})$ & 0.00125 \\
flow rate of supplied debris flow per unit width $\left(\mathrm{cm}^{2} / \mathrm{s}\right)$ & 127.3 \\
sediment concentration of supplied debris flow & 0.349 \\
degree of upper stream $\left(^{\circ}\right)$ & 18.0 \\
degree of lowe stream $\left(^{\circ}\right)$ & 4.0 \\
density of water, $\rho_{w}$ & 1.00 \\
density of grains, $\sigma$ & 2.65 \\
friction angle, $\varphi_{\mathrm{s}}\left({ }^{\circ}\right)$ & 38.5 \\
sediment concentration of sediment layer, $c *$ & 0.60 \\
coefficient of restitution, $e$ & 0.78 \\
average diameter, $d(\mathrm{~cm})$ & 0.342 \\
interparticle distance, $l_{0}(\mathrm{~cm})$ & 0.25 \\
\hline
\end{tabular}

\begin{tabular}{l|c|c}
\hline & Case2 & Case3 \\
\hline relaxation time, $T(\mathrm{~s})$ & 0.0005 & 0.0002 \\
flow rate of supplied water $\left(\mathrm{cm}^{3} / \mathrm{s}\right)$ & 500 & 2000 \\
supplied sediment volume $\left(\mathrm{cm}^{3}\right)$ & 2000 \\
degree of channel $\left({ }^{\circ}\right)$ & 11.3 \\
density of water, $\rho_{w}$ & 1.00 \\
density of grains, $\sigma$ & 2.65 \\
friction angle, $\varphi_{\mathrm{s}}\left(^{\circ}\right)$ & 30.0 \\
sediment concentration of sediment layer, $c *$ & 0.60 \\
coefficient of restitution, $e$ & 0.78 \\
average diameter, $d(\mathrm{~cm})$ & 0.44 \\
interparticle distance, $l_{0}(\mathrm{~cm})$ & 0.10 \\
\hline
\end{tabular}

$$
\frac{\partial h}{\partial t}+\frac{\partial M}{\partial x}=E
$$

and the continuity equation for the particles is

$$
\frac{\partial(\bar{c} h)}{\partial t}+\frac{\partial\left(c_{t} M\right)}{\partial x}=E c_{*}
$$

Changes in the bed surface elevation can be determined using

$$
\frac{\partial z_{b}}{\partial t}=-E
$$

In Eqs. (28)-(31), $h$ is the flow depth, $u$ is the flow velocity, $M=u h, g$ is the acceleration due to gravity, $\rho_{m}$ is the density of the debris flow, $H=h+z_{b}, z_{b}$ is the bed elevation, $E$ is the erosion rate, $\tau_{0}$ is the riverbed shear stress, $\bar{c}(Z)$ is the average sediment concentration, and $c_{t}$ is the flux sediment concentration.

The equations of Miyamoto and Itoh [2002] were applied to calculate $\tau_{0}$ and $c_{t}$, and the following erosion rate equation of Suzuki et al. [2009] was applied for $E$ :

$$
E=\frac{1}{T c_{*}}\left(h_{e} c_{e}-h \bar{c}\right)
$$

where $T$ is the relaxation time for erosion or deposition, and $h_{e}$ is the equilibrium flow depth when $\tau_{0}$ is equal to the external force.

\section{CONDITIONS FOR NUMERICAL SIMULATION}

\subsection{Deposition process}

Numerical simulations were carried out under the conditions of the flume experiment described by Takahama et al. [2002], where the gradient of the riverbed changes abruptly from $18^{\circ}$ to $4^{\circ}$. The bed was rigid, and the sediment particles were located to describe the roughness. Water and sediment were regularly supplied from the upper end. The flow rate per unit width of supplied debris flow was 128.0 $\mathrm{cm}^{2} / \mathrm{s}$, the sediment concentration was 0.306 , and the sediment diameter was $0.342 \mathrm{~cm}$ (Case 1). The parameters for numerical simulation are listed in Table 1.

\subsection{Erosion process of natural landslide dam}

Numerical simulations were carried out under the conditions of the flume experiments reported by Oda et al. [2010]. The channel width was $15 \mathrm{~cm}$ and the gradient of the riverbed was $11.3^{\circ}$. Water was supplied from the upper end and sediment was supplied to the passing water. The flow rate of supplied water was $250 \mathrm{~cm}^{3} / \mathrm{s}$ (Case 2) and 1000 $\mathrm{cm}^{3} / \mathrm{s}$ (Case 3), the volume of supplied sediment was $2000 \mathrm{~cm}^{3}$, and the average sediment diameter was $0.44 \mathrm{~cm}$. Oda et al. [2010] examined the temporal variation in the shape of landslide dams and the erosion rate around the crown of the landslide dam; however, only the erosion process was considered in this study. Therefore, the shapes of the landslide dams at the onset of overtopping were applied as the initial conditions for the numerical simulations. The parameters used in the numerical simulations are listed in Table $\mathbf{1}$.

\subsection{Relaxation time}

A long relaxation time, $T$, corresponds to highly non-equilibrium dynamics. In this study, $T$ was set to be significantly lower than the time required for moving $l_{0}$ with the maximum velocity, such that the local equilibrium of the sediment concentration was nearly satisfied [Suzuki and Hotta, 2015] (Table 1).

\section{RESULTS}

\subsection{Deposition process}

Figure 3 shows a comparison of the experimental results reported by Takahama et al. [2002] and our simulated data for the surface profiles of the riverbed and the flow. The coordinates were changed so that the lower stream was horizontal. The time since the front of debris 


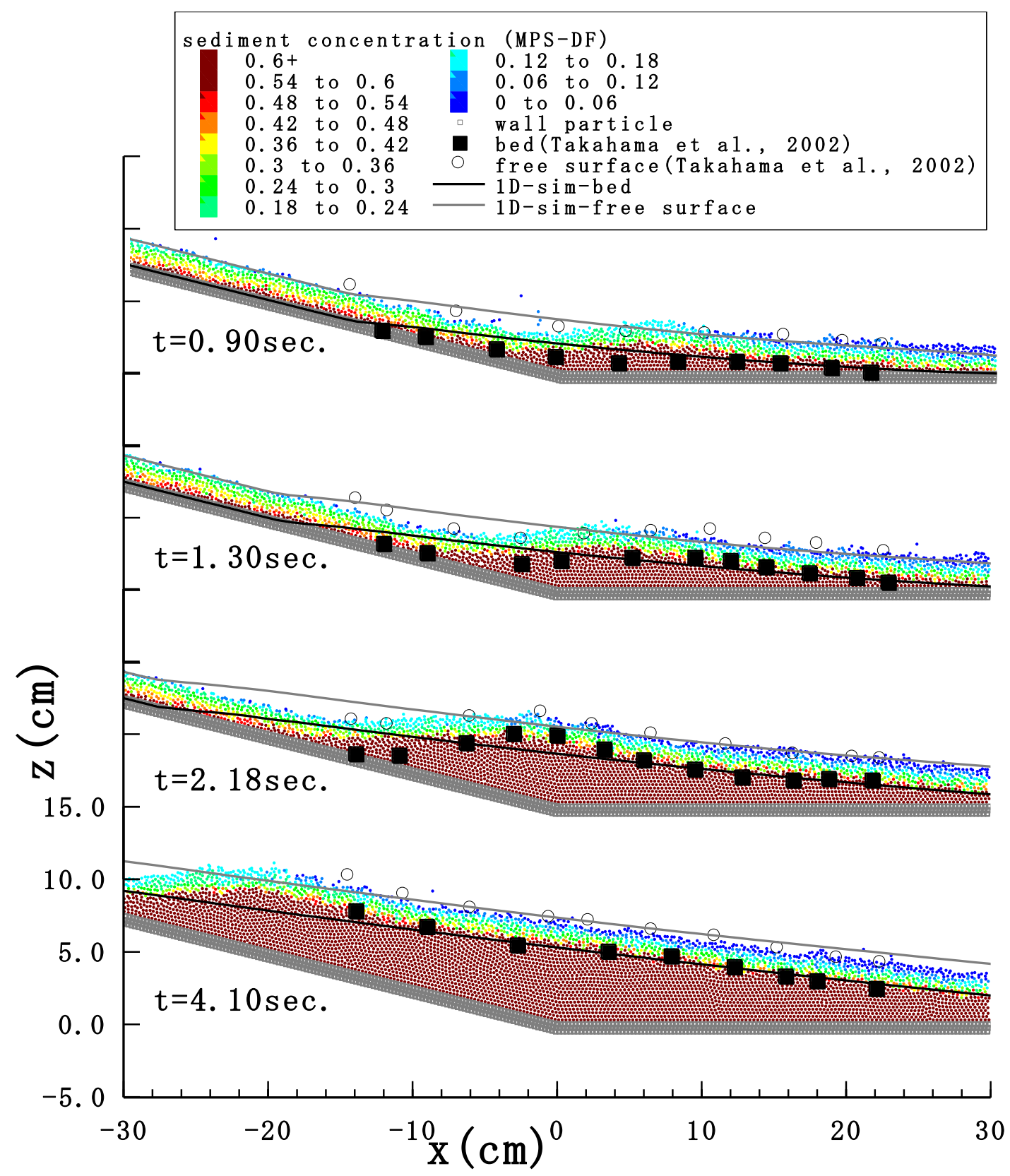

Fig. 3 Simulated data and experimental results at the point at which the gradient of the riverbed changes (Case 1).

flow reached the point at which the gradient of the riverbed changes is shown in Fig. 3. With the MPS-DF model, when the particles move to a region with a smaller low gradient, $c$ moves closer to the riverbed particles, and the riverbed particles stop. This is because the shear stress equation of Egashira et al. [1989] contains a Coulomb friction term. As described above, particles with a sediment concentration larger than $0.9 c *$ were considered to be deposited. In addition, when the low $c$ particles move over riverbed particles, $c$ moves from the riverbed particles to the flow particles, and the riverbed particles start to move. This process corresponds to the erosion.

After $4.1 \mathrm{~s}$, the experimental results, as well as the simulated results obtained using both $1 \mathrm{D}$-sim and MPS-DF were in good agreement. The experimental results show a convex upward deposition shape during the early stages of the process, and a run-up to the upper stream occurred as time progressed. The $1 \mathrm{D}$-sim method could not reproduce this process; however the MPS-DF method was able to reproduce a very similar result.

\subsection{Erosion process of natural landslide dam}

Figure 4 shows the simulation results for Case 2 and Fig. 5 those for Case 3. In both figures, the experimental results of Oda et al. [2010] are also shown for comparison. The time since overtopping began is shown, and the coordinates were modified 


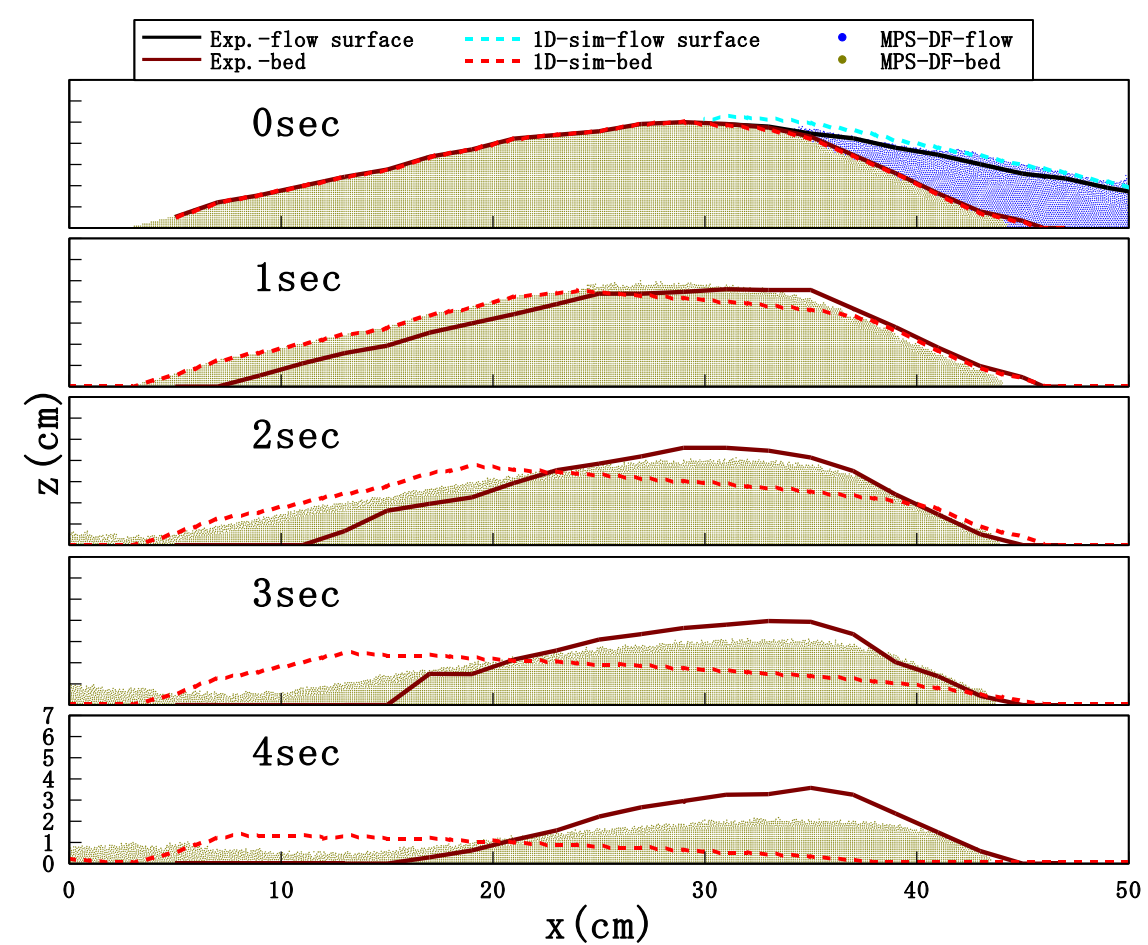

Fig. 4 Simulated data and experimental results of the erosion process of the landslide dam (Case 2).

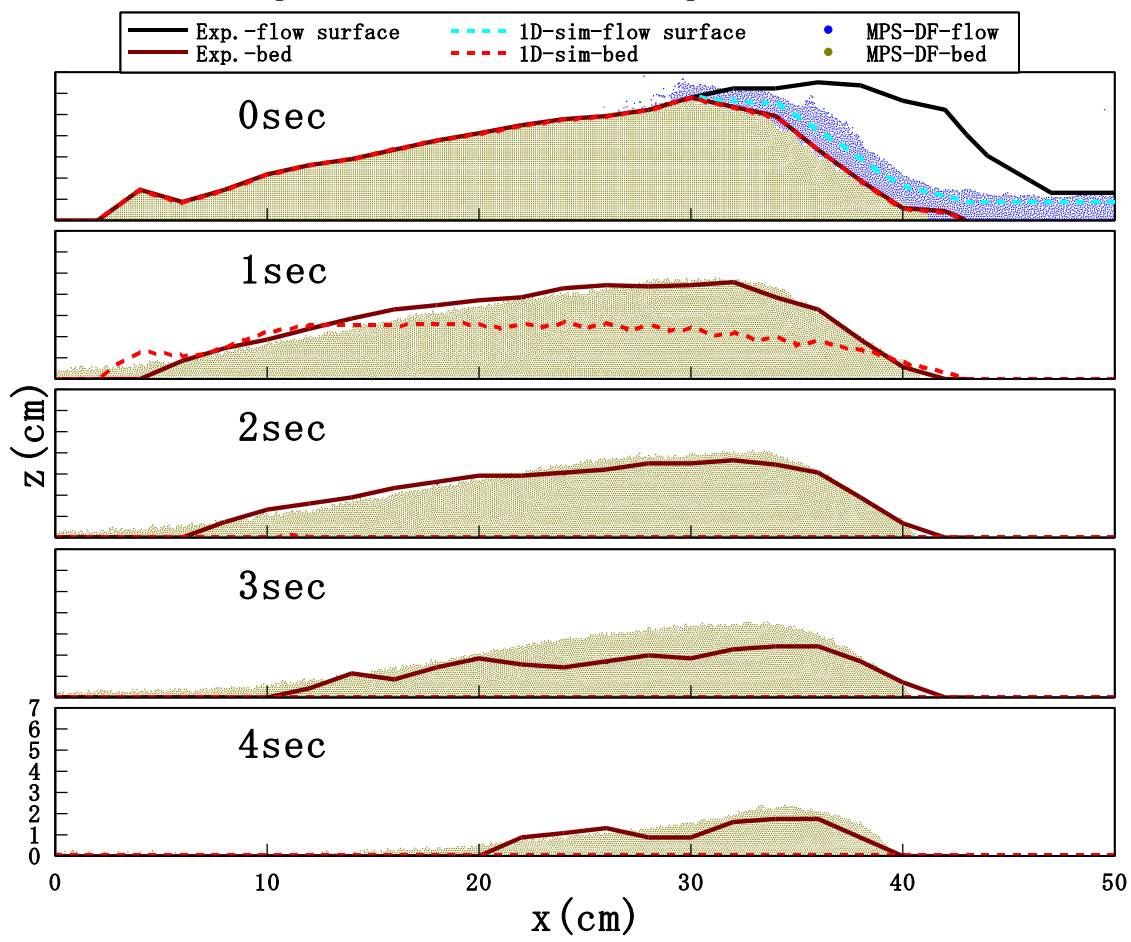

Fig. 5 Simulated data and experimental results of the erosion process of the landslide dam (Case 3).

so that the riverbed was horizontal.

With 1D-sim, the erosion rate around the crown was much more rapid than with either MPS-DF or the experimental results in both cases. Furthermore, 1D-sim resulted in deposition of eroded sediment at the lower side of the landslide dam, which did not occur in the experimental results. The erosion processes with the MPS-DF method provided good agreement with the experimental results; however, the erosion rate with the MPS-DF method was slightly faster than the experimental results for Case 2. In addition, MPS-DF resulted in deposition at the lower side of the landslide with Case 2; however, the amount of deposition was much smaller than with 1D-sim. 


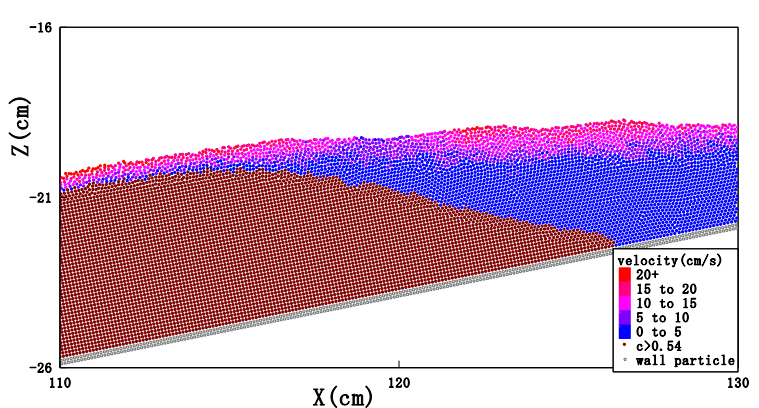

Fig. 6 The vertical distribution of the flow velocity during the initial stages of the process of overtopping (Case 2).

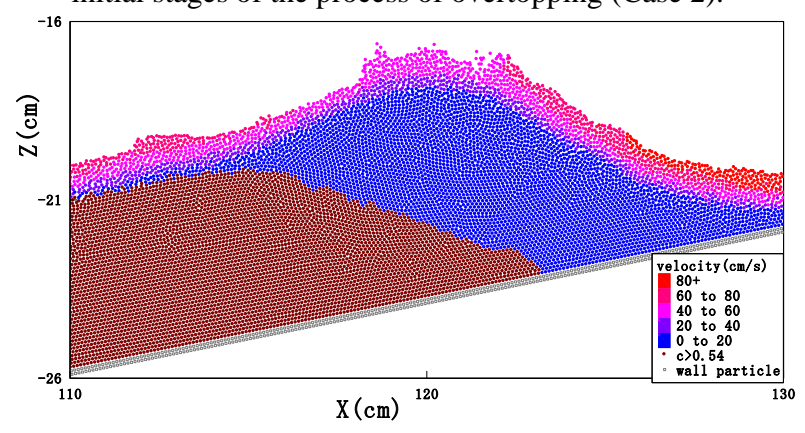

Fig. 7 The vertical distribution of the flow velocity during the initial stages of the process of overtopping (Case 3).

\section{DISCUSSION AND CONCLUSION}

All numerical simulations were carried out with the conditions that local equilibrium of sediment concentration was almost satisfied. In deposition processes at the location of the change of gradient of the riverbed, the results of the $1 \mathrm{D}$-sim method did not provide good agreement with the experimental results during the early stages of the process. In contrast, the results obtained using the MPS-DF method reproduced a convex upward deposition shape during the early stages of the process. The reason for this is thought to be the difference in the methods of evaluating the energy gradient. With the 1D-sim method, the riverbed gradient was included as a cross-sectional parameter, whereas the flow gradient of each particle was used with the MPS-DF method. In other words, the MPS-DF method can be used to calculate the energy gradient accurately when the upper and lower layers have different flow directions. For this reason, we expect that the MPS-DF method will provide better accuracy during the early stages of the deposition process. When the deposition advanced, the energy gradient became almost equal to the riverbed gradient, and the 1D-sim method provided high accuracy as MPS-DF method. Moreover, 1D-sim is based on a coordinate system perpendicular to the riverbed. Axis directions vary widely around a sharp change in the riverbed gradient. This may have reduced the accuracy in 1D-sim.

During erosion of a natural landslide dam, the erosion rate with the 1D-sim method was much faster than the experimental results. The erosion rate was calculated using the present sediment concentration and the equilibrium sediment concentration corresponding to the riverbed gradient (i.e., Eqs. (27) and (32)). During the erosion process, the onset of movement of grains occurs near the riverbed; however, the 1D-sim method resulted in sediment concentration that approached equilibrium instantaneously, because of the requirement that local equilibrium is satisfied. In other words, with the 1D-sim, at some depth into the sediment layer, an abrupt transition between moving and stationary layers occurs, which results in an overestimation of the erosion rate. In contrast, with the MPS-DF method, the sediment concentration can vary among neighboring particles. Therefore, only neighboring particles in the flow layer contribute to the erosion process. We expect that the MPS-DF method is able to better reproduce the erosion process for this reason.

Vertical averaging of the flow velocity is considered to be another reason for inaccuracies with 1D-sim. The cross-sectional averaged flow is used with this method based on the shallow water equation, and therefore all of the impounded water of the landslide dam has the same velocity. In contrast, the MPS-DF method can reproduce the distribution of flow velocity. Figure 6 (Case 2) and Fig.7 (Case 3) show the distribution of the flow velocity during the initial stages of the process of overtopping. The major part of overflow water originated from the upper layer of the impound water, and was in good agreement with the experimental observations.

The results discussed above show that an exact evaluation of the energy gradient and local equilibrium of the sediment concentration are necessary for accurate numerical simulations. However, the MPS-DF method resulted in the smallest deposition at the lower side of the landslide dam in Case 2. The reasons for this are unclear; however, improvements in the calculation method including the boundary conditions and more detailed modeling of multi-granular debris flows [Nishiguchi et al., 2011; Iwata et al., 2013] may be beneficial as part of further work.

ACKNOWLEDGMENT: The authors wish to thank Prof. A. Oda, Nihon University, Japan, for provision of experimental data.

\section{REFERENCES}

Abe, T., Satofuka, Y. and Mizuyama, T. (2011): Development of modified particles method for simulations of 
highly-concentrated granular flows considering inter-particle stress, Journal of the Japan Society of Erosion Control Engineering, Vol. 63, No. 6, pp. 23-31 (in Japanese with English abstract).

Egashira, S., Ashida, K., Yajima, H. and Takahama, J. (1989): Constitutive equations of debris flow, Annuals, Disas. Prev. Res. Inst., Kyoto Univ., No. 32B-2, pp. 487-501 (in Japanese with English abstract).

Egashira, S., Miyamoto, K. and Itoh, H. (1997): Constitutive equations of debris flow and their applicability, Proc. 1st International Conference on Debris-Flow Hazards Mitigation, ASCE, pp. 340-349.

Iwata, T., Hotta, N. and Suzuki, T. (2013): Influence of particle-size segregation in multi-granular debris flows on the fluidity, Journal of the Japan Society of Erosion Control Engineering, Vol. 66, No. 3, pp. 13-23 (in Japanese with English abstract).

Khayyer, A. and Gotoh, H. (2008): Development of CMPS method for accurate water-surface tracking in breaking waves, Coastal Engineering Journal, Vol. 50, No. 2, pp. 179-207.

Koshizuka, S., Tamako, H. and Oka, Y. (1995): A particle method for incompressible viscous flow with fragmentation, Comput. Fluid Dyn. J., Vol. 4, No. 1, pp. 29-46.

Koshizuka, S. and Oka, Y. (1996): Moving particle semi-implicit method for fragmentation of incompressible fluid, Nuclear Science and Engineering, Vol. 123, pp. 421-434.

Minatti, L. and Pasculli, A. (2011): SPH numerical approach in modeling 2D muddy debris flow, In Proc. Symposium on Computer Animation (2003), pp.154-159.

Miyamoto, K. and Itoh, T. (2002): Numerical simulation method of debris flow introducing the erosion rate equation, Journal of the Japan Society of Erosion Control Engineering, Vol. 55, No. 2, pp. 24-35 (in Japanese with English abstract).

Muller, M., Charypar, D. and Gross, M. (2003): Particle-based fluid simulation for interactive applications, Italian Journal of Engineering Geology and Environment - Book, pp. 467-475.

Nakagawa, H., Takahashi, T., Satofuka Y. and Kawaike, K. (2001): Sediment disasters caused by the heavy rainfall in the Camri Grande River basin, Venezuela, 1999 Reproduction of sediment runoff, flooding, and deposition and evaluation of effectiveness of the sabo works by means of numerical simulation -, Annuals, Disas. Prev. Res. Inst., Kyoto Univ., Vol. 44, No. B-2, pp. 207-228 (in Japanese with English summary).

Nishiguchi, Y., Uchida, T., Ishizuka, T., Satofuka, T. and Nakatani, K. (2011): Numerical simulation for run out process of large-scale debris flow focused on fine sediments behaviors : application for debris flow triggered by a deep catastrophic landslide, Journal of the Japan Society of Erosion Control Engineering, Vol. 64, No. 3, pp. 11-20 (in Japanese with English abstract).

Oda, A., Mizuyama, T. and Miyamoto, K. (2010): Experimental study of the shape of small landslide dams and the hydrograph during the outburst of small landslide dams, Journal of the Japan Society of Erosion Control Engineering, Vol. 63, No. 3, pp. 3-10 (in Japanese with English abstract).

Satofuka, Y., Yoshino, K., Ogawa, K. and Mizuyama, T. (2007): Prediction of flood peak discharge at landslide dam outburst, Journal of the Japan Society of Erosion Control Engineering, Vol. 59, No. 6, pp. 55-59 (in Japanese with English abstract).

Suzuki, T., Hotta, N. and Miyamoto, K. (2009): Numerical simulation method of debris flow introducing the non-entrainment erosion rate equation, at the transition point of riverbed gradient or the channel width and in the area of sabo dam, Journal of the Japan Society of Erosion Control Engineering, Vol. 62, No. 3, pp. 14-22 (in Japanese with English abstract).

Suzuki, T. and Hotta, N. (2015): Development of modified particles method for simulations of debris flows based on constitutive equations and its application to deposition process, Journal of the Japan Society of Erosion Control Engineering, Vol. 68, No. 1, pp. 13-24 (in Japanese with English abstract).

Takahama, J., Fujita, Y., Hachiya, K. and Sakamoto, M. (2002): Study on debris flow behavior in a region around a point of abrupt slope reducing, Annual Journal of Hydraulic Engineering, JSCE, Vol. 46, pp. 683-688 (in Japanese with English abstract).

Received: 15 July, 2015

Accepted: 8 March, 2016 